\title{
SEGMENTATION-BASED GROUND POINTS DETECTION FROM MOBILE LASER SCANNING POINT CLOUD
}

\author{
Xiangguo Lin ${ }^{\text {a, } * \text {, Jixian Zhang }}{ }^{\text {a }}$ \\ ${ }^{\text {a }}$ Institute of Photogrammetry and Remote Sensing, Chinese Academy of Surveying and Mapping, Beijing 100830, China- \\ linxiangguo@gmail.com; zhangjx@casm.ac.cn
}

Commission VII, WG VII/6

KEY WORDS: Filtering, Mobile Laser Scanning, Point Cloud, Point Cloud Segmentation

\begin{abstract}
:
In most Mobile Laser Scanning (MLS) applications, filtering is a necessary step. In this paper, a segmentation-based filtering method is proposed for MLS point cloud, where a segment rather than an individual point is the basic processing unit. Particularly, the MLS point cloud in some blocks are clustered into segments by a surface growing algorithm, then the object segments are detected and removed. A segment-based filtering method is employed to detect the ground segments. Two MLS point cloud datasets are used to evaluate the proposed method. Experiments indicate that, compared with the classic progressive TIN (Triangulated Irregular Network) densification algorithm, the proposed method is capable of reducing the omission error, the commission error and total error by $3.62 \%, 7.87 \%$ and $5.54 \%$ on average, respectively.
\end{abstract}

\section{INTRODUCTION}

In the last decade, mobile laser scanning (MLS) is a quite new technology in which environment are mapped by laser distance measurements from moving vehicles, and transformed into a georeferenced 3D point cloud using GPS/IMU data. As a stateof-the-art technology for mapping and remote sensing, MLS can serve as an effective solution for surveying complex environment, such as urban environment and road corridors (Lin et al., 2013). Many mature MLS systems can be found from the market (Kaartinen et al., 2012), and widely used for various purposes such as road inventory (Pu et al., 2011), map update (Hwang et al., 2013), façade extraction (Yang et al., 2013; Jochem et al., 2011), building reconstruction (Frueh et al., 2005; Becker et al., 2009), road marking extraction (Yang et al., 2012), window extraction (Wang et al., 2012), tree extraction (Wu et al., 2013), object extraction and recognition (Yang et al., 2012; Yu et al., 2013; Golovinskiy et al., 2009, etc).

In fact, among various applications, detection of ground points is a necessary step. Because the ground points probably make up the largest percentage of entire points and the objects of interest are usually located on the ground surface $(\mathrm{Pu}$ et al., 2011; Golovinskiy et al., 2009; Elhinney et al., 2010). However, the existing ground detection methods for airborne LiDAR point cloud are also employed for MLS point cloud, and they are faced with the following problems:

The huge amount of points (Pu et al., 2011; Yang et al., 2013) causes heavy computational burden. Thus, object points should be partially removed before filtering.

Existence of outliers, especially low outliers, may lead to many errors (Elhinney et al., 2010). Thus, the outliers should be eliminated at first for most filtering methods.

There are variable point densities (Yang et al., 2013) and data gap, which may make the ground surface not well sampled.

A point cloud is normally composed of various types of complex and incomplete scene structures (Yang et al., 2013), and the lower parts of off-terrain objects (such as vehicle, façade, tree trunk, etc.) are attached on the ground surface, thus the points belonging to the lower parts are likely to being classified as ground measurements.

Urban ground surface itself is not smooth and continuous enough, because there are lots of break lines such as road edges and curbstones (Zhou et al., 2012). However, the points around the break lines may be misclassified.

Practices suggest filtering of LiDAR data can be strengthened by analyzing segments rather than individual points (Filin et al., 2006). Moreover, once a point cloud has been segmented, segment attributes can be collected to classify the segments (Vosselman et al., 2010). As a result, similar to object-based image analysis (Blaschke et al., 2010), a segment-based classification is more reliable than a point-based classification (Vosselman et al., 2010; Zhang et al., 2013; Rutzinger et al., 2008) for point cloud. Thus, a segment-based method for ground measurement detection from MLS point cloud is proposed herein. The main contribution of our method consists of two parts. The first one is a knowledge-based method ( $\mathrm{Pu}$ et al., 2011) is employed to detect the off-terrain objects. The second one is a segment-based method for point cloud filtering is proposed to remove the lower parts of objects and retain the points around break lines.

\footnotetext{
* Corresponding author
} 


\section{METHOD}

Our proposed method is composed of four core steps, namely data partition, point cloud segmentation, object segments detection, and segment-based progressive TIN densification (PTD). Moreover, the classic point-based PTD method (Axelsson, 2000) is widely employed in filtering of point cloud, and it is improved by our segment-based method.

\subsection{Data partition}

Processing MLS point cloud is a challenging task due to the huge point data volumes. Instead of processing the whole point cloud directly, the raw MLS point cloud are partitioned into multiple blocks, and then information of interest is extracted from the points in each block,. The geometrical shape of each block is manually specified, and the overlapping zones between neighbouring blocks can be permitted. After data partition, each dataset in a block is taken as an independent point cloud taking part in the following processing.

\subsection{Point cloud segmentation}

Point cloud segmentation is the process to partition a point cloud into coherent and connected point clusters. Specifically, points on a certain geometric feature are coherent points, such as co-plane, co-surface, and co-line points; whilst connected points are a group of points in which every point has at least one neighbouring point within a certain distance.

The surface growing algorithm proposed by Vosselman and Klein (2010) is used herein. After data partition, the laser point cloud in each block is segmented into planar segments with a surface growing algorithm. After segmentation, the ground surface is clustered into several large segments, each building façade may be clustered into one segment, and the vegetation has many small segments. Moreover, the surface growing method supposes that some points are outliers, thus they are not labeled in segmentation. These outliers are eliminated from the point cloud, and only the planar segments are used for the following operations.

\subsection{Object segments detection}

Geometric features of a segment and topological relations between segments have been widely employed in information extraction from point cloud ( $\mathrm{Pu}$ et al., 2011). Generally, most building façade segments are very large and aligned vertically (Yang et al., 2013; Jochem et al., 2011), and most vegetation segments are small (Zhang et al., 2013; Rutzinger et al., 2008) and scattered in 3D space (Yang et al., 2013; Zhang et al., 2013).Two features about orientation and scatterness are selected to detect object segment herein. A method to calculate the orientation and the scatterness of a planar segment was proposed in (Zhang et al., 2013), where the scatterness is calculated based on the principal components analysis (Yang et al., 2013). Based on visual evaluation of the histograms of orientation and scatterness, two thresholds about orientation $O$ and scatterness $S$ for distinguishing the façade and nonfaced, vegetation and non-vegetation can be determined in a tryand-error way. In our proposed approach, the potential building façade segments and vegetation segments are first detected and labeled as "object"; and they are eliminated from the following processing, which will reduce the volume of points for the subsequent step.

\subsection{Segment-based PTD}

This step is similar to the PTD filter, but the basic processing unit is a segment rather than a single point. It is composed of five core steps, and details of five steps refer to Section 3.4 in (Lin et al., 2014).

\section{EXPERIMENTS AND PERFORMANCE EVALUATION}

\subsection{Test data and the relevant parameters}

Two test datasets were acquired using the SSW mobile mapping system. The SSW mobile laser scanning system is developed and made by Beijing 4D Vision Information Technology Co. Ltd, China. The hardware of the SSW consists of a laser scanner, a CCD camera system, a portable control unit box, a GNSS/INS unit etc. The laser scanner collects data at a rate of 200,000 measurements per second with a field of view (FOV) of $360^{\circ}$. The first data has 2,000,173 points, with an average point density of 110 points $/ \mathrm{m}^{2}$; while the second data has $2,000,055$ points, with an average point density of 160 points $/ \mathrm{m}^{2}$. The first data covers an area in Beijing City, China, with approximate $350 \mathrm{~m}$ length and $500 \mathrm{~m}$ width. The second data covers an area in Sanxia City, China, with approximate $330 \mathrm{~m}$ length and $220 \mathrm{~m}$ width. Moreover, both datasets have various types of artificial objects and the ground surfaces are not smooth enough.

The shared five parameters $m, t, \theta, d, l$ are set to the same values for both filters applied on the two datasets, as shown in Table 1. Moreover, our proposed method needs more parameters. Specifically, in the surface growing segmentation, the orientation threshold, $o$, and scatterness threshold, $s$ are set to $45^{\circ}$ and 0.2 respectively for both two data.

\subsection{Results and Performance Evaluation}

With the specified parameters in Table 1, we perform the filtering on the two datasets using the two methods.

Visual inspection suggests that the surface growing method yields good results, and there is less under-segmentation and over-segmentation. Some statistics about the filtering results refer to Table 2. For the PTD method, there are 57 outliers and 718 outliers in the two test datasets, respectively. 64 points and 60 points are selected as seed points in the two test datasets; there are 1,035,516 ground points and 1,155,869 ground points in the final filtered results. For our proposed method, there are 974,043 object points and 1,205,793 object points detected by the step in the Section 3.3 in the two test datasets; there are 535,226 points and 900,949 points selected as seed points in the two test datasets; there are 994,760 ground points and $1,135,437$ ground points in the final filtered results. The statistics in Table 2 suggest that our method does not need outlier removal step, it is capable of detecting most of the object measurements, and it is capable of selecting many more seed ground points.

Quantitative assessment follows the method proposed in ISPRS filter test (Sithole et al., 2004). Three kinds of errors are calculated, namely, type I errors (i.e., omission errors), type II errors (i.e., commission errors), and total errors. Moreover, the reference results of the two datasets are produced in a way of combination of automatic filtering and manual editing. The three types of errors of the two filters for the two datasets are 
listed in Table 3. For our proposed method, the three types of errors are $0.07 \%, 0.39 \%$ and $0.23 \%$ for the first test dataset; the three types of errors are $4.74 \%, 0.09 \%$ and $2.86 \%$ for the second test dataset. For the PTD method, the three types of errors are $3.23 \%, 7.55 \%$ and $5.40 \%$ for the first test dataset; the three types of errors are $8.85 \%, 8.66 \%$ and $8.77 \%$ for the second test dataset. The statistics in Table 3 suggest that our proposed approach achieve better results than the classic PTD method. On average, compared with the PTD algorithm, the type I error, the type II error and total error of our method are reduced by $3.62 \%, 7.87 \%$ and $5.54 \%$. Moreover, in both cases, the three types of errors of our method are quite low, which shows that our filtered results are very close to the ground truth data.

\begin{tabular}{|l|l|l|l|l|l|}
\hline Parameters & $m_{(\mathrm{m})}$ & $t_{\left({ }^{\circ}\right)}$ & $\theta_{\left({ }^{\circ}\right)}$ & $d_{(\mathrm{m})}$ & $l_{(\mathrm{m})}$ \\
\hline $\begin{array}{l}\text { Threshold } \\
\text { value }\end{array}$ & 60 & 88 & 6 & 0.5 & 1.0 \\
\hline
\end{tabular}

Table 1. Input five shared parameters of the two filters in the two datasets

\begin{tabular}{|c|c|c|c|c|c|c|c|}
\hline \multirow[t]{2}{*}{ Indices } & \multirow{2}{*}{$\begin{array}{l}\text { Total } \\
\text { number } \\
\text { of points } \\
\text { (points) }\end{array}$} & \multicolumn{3}{|c|}{ Classic PTD method } & \multicolumn{3}{|c|}{ Our method } \\
\hline & & $\begin{array}{l}\text { Number } \\
\text { of } \\
\text { outliers } \\
\text { (points) }\end{array}$ & $\begin{array}{l}\text { Number } \\
\text { of seed } \\
\text { points } \\
\text { (points) }\end{array}$ & $\begin{array}{l}\text { Number } \\
\text { of ground } \\
\text { points } \\
\text { (points) }\end{array}$ & $\begin{array}{l}\text { Number } \\
\text { of object } \\
\text { points } \\
\text { (points) }\end{array}$ & $\begin{array}{l}\text { Number } \\
\text { of seed } \\
\text { points } \\
\text { (points) }\end{array}$ & $\begin{array}{l}\text { Number } \\
\text { of ground } \\
\text { points } \\
\text { (points) }\end{array}$ \\
\hline $\begin{array}{l}\text { Data } \\
\text { set } 1\end{array}$ & $2,000,173$ & 57 & 64 & $1,035,516$ & 974,043 & 535,226 & 994,760 \\
\hline $\begin{array}{l}\text { Data } \\
\text { set } 2\end{array}$ & $2,000,055$ & 718 & 60 & $1,155,869$ & $1,205,793$ & 900,949 & $1,135,437$ \\
\hline
\end{tabular}

Table 2. Statistics about the filtered results of the two filters

The experiments indicate that our proposed method has quite better performances than the classic PTD method. Our method's advantages come from the embedding of point cloud segmentation, which makes the judging in a segment-wise manner. In the segment-based judging, the lower parts of the off-terrain objects are less possible being detected as ground, while the points around the road edges are more possible being detected as ground.

\begin{tabular}{|c|c|c|c|}
\hline Dataset NO. & Type of error & $\operatorname{PTD}(\%)$ & Our method(\%) \\
\hline \multirow{3}{*}{ Data set 1} & I & 3.23 & 0.07 \\
\hline & II & 7.55 & 0.39 \\
\hline & $\mathrm{T}$ & 5.40 & 0.23 \\
\hline \multirow{3}{*}{ Data set 2} & I & 8.85 & 4.74 \\
\hline & II & 8.66 & 0.09 \\
\hline & $\mathrm{T}$ & 8.77 & 2.86 \\
\hline
\end{tabular}

Table 3. Three types of errors of the two filters in the two test datasets

\section{CONCLUSIONS AND DISCUSSION}

Filtering is one of the core post-processing steps for MLS point cloud. However, the classic PTD filter fails to remove the lower parts of the objects and preserve the ground measurements in steep terrain areas. Thus, a segment-based filtering method is proposed by integrating the PTD framework and surface growing segmentation method. The experiments are performed on two datasets to verify our proposed method; moreover, two ground truth datasets are produced to calculate the accuracies. The results suggest that, our proposed approach is better than the classic PTD method in removing vehicle measurements and preserving ground measurements. Moreover, our approach solves the five problems of the PTD method listed in Section 1. Particularly, it has significantly lower type I errors, type II errors and total errors than the PTD algorithm. The future work will focus on the improvement of the proposed filter to reduce the type II error, and parallel computing is going to be implemented to promote the efficiency.

\section{ACKNOWLEDGEMENTS}

This research was funded by the General Program sponsored by the National Natural Science Foundations of China (NSFC) under Grant 41371405.

\section{REFERENCES}

Lin, Y., Hyyppä, J., Kaartinen, H. and Kukko, A., 2013. Performance analysis of mobile laser scanning systems in target representation. Remote Sensing, 5 (7), pp. 3140-3155.

Kaartinen, H., Hyyppä, J., Kukko, A., Jaakkola, A and Hyyppä, H., 2012. Benchmarking the performance of mobile laser scanning systems using a permanent test field. Sensors, 12 (9), pp. 12814-12835.

$\mathrm{Pu}$, S. Rutzinger, M. Vosselman, G and Elberink, S. Oude., 2011. Recognizing basic structures from mobile laser scanning data for road inventory studies. ISPRS Journal of Photogrammetry and Remote Sensing, 66 (6), pp. S28-S39.

Hwang, J., Yun, H., Jeong, T., Suh, Y and Huang, H., 2013. Frequent unscheduled updates of the national base map using the land-based mobile mapping system. Remote Sensing, 5 (5), pp. 2513-2533.

Yang, B., Wei, Z., Li, Q and Li, J., 2013. Semiautomated building facade footprint extraction from mobile lidar point cloud. IEEE Geoscience and Remote Sensing Letters, 10 (4), pp. 766-770. 
Jochem, A., Höfle, B and Rutzinger, M., 2011. Extraction of vertical walls from mobile laser scanning data for solar potential assessment. Remote Sensing, 3 (4), pp. 650-667.

Frueh, C., Jain, S and Zakhor, A., 2005. Data processing algorithms for generating textured 3D building façade meshes from laser scans and camera images. International Journal of Computer Vision, 61 (2), pp. 159-184.

Becker, S. and Haala, N., 2009. Grammar supported facade reconstruction from mobile lidar mapping. Internationa Archives of Photogrammetry, Remote Sensing and Spatial Information Sciences, 38 (3w4), pp. 229-234.

Yang, B., Fang, L., Li, Q and Li, J., 2012. Automated extraction of road markings from mobile Lidar point cloud. Photogrammetric Engineering \& Remote Sensing, 78 (4), pp. 331-338.

Wang, R., Ferrie F and Macfarlane, J., 2012. A method for detecting windows from mobile LiDAR data. Photogrammetric Engineering and Remote Sensing, 78 (11), pp.1129-1140.

Wu, B., Yu, B., Yue, W., Shu, S., Tan, W., Hu, C., Huang, Y., Wu J., and Liu, H., 2013. A voxel-based method for automated identification and morphological parameters estimation of individual street trees from mobile laser scanning data. Remote Sensing, 5 (2), pp.584-611.

Yang, B., Wei, Z and Li, Q., 2012. Automated extraction of street-scene objects from mobile lidar point cloud. International Journal of Remote Sensing, 33 (18), pp. 5839-5861.

Yu, Y., Li, J., Yu, J and Wang, C., 2013. Pairwise threedimensional shape context for partial object matching and retrieval on mobile laser scanning data. IEEE Geoscience and Remote Sensing Letters, 11 (5), pp. 1019-1023.

Golovinskiy, A., Kim, V. G and Funkhouser, T., 2009. Shapebased recognition of 3D point cloud in urban environments. In: 12th International Conference on Computer Vision (ICCV), IEEE, pp. 2154-2161.

Elhinney, C. Mc., Kumar, P., Cahalane, C and McCarthy, T., 2010. Initial results from European road safety inspection (EuRSI) mobile mapping project. International Archives of Photogrammetry, Remote Sensing and Spatial Information Sciences, 38 (5), pp. 440-445.

Yang, B and Dong, Z., 2013. A shape-based segmentation method for mobile laser scanning point cloud. ISPRS Journal of Photogrammetry and Remote Sensing, 81, pp. 19-30.

Zhou, L and Vosselman, G., 2012. Mapping curbstones in airborne and mobile laser scanning data. International Journal of Applied Earth Observation and Geoinformation, 18, pp. 293-304.

Filin, S. and Pfeifer, N., 2006. Segmentation of airborne laser scanning data using a slope adaptive neighbourhood. ISPRS Journal of Photogrammetry and Remote Sensing, 60 (2), pp. 71-80. Apr. 2006

Vosselman, G and Klein, R., 2010. Visualization and structuring of point cloud. In: Airborne and Terrestrial Laser Scanning, 1st ed.; Vosselman, G., Maas, H.G., Eds.; Whittles Publising: Dunbeath, pp. 43-79.
Blaschke, T., 2010. Object based image analysis for remote sensing. ISPRS Journal of Photogrammetry and Remote Sensing, 65 (1), pp. 2-16.

Zhang ,J., Lin, X and Ning, X., 2013. SVM-based classification of segmented airborne LiDAR point cloud $\mathrm{s}$ in urban areas. Remote Sensing, 5 (8), pp. 3749-3775.

Rutzinger, M., Höfle, B., Hollaus, M and Pfeifer, N., 2008. Object-Based point cloud analysis of full-waveform airborne laser scanning data for urban vegetation classification. Sensors, 8 (8), pp. 4505-4528.

Sithole, G. and Vosselman, G., 2004. Experimental comparison of filter algorithms for bare earth extraction from airborne laser scanning point cloud. ISPRS Journal of Photogrammetry and Remote Sensing, 59 (1-2), pp. 85-101.

Lin, X., Zhang, J., 2014. Segmentation-based filtering of airborne LiDAR point clouds by progressive densification of terrain segments. Remote Sensing, 6(2), pp. 1294-1326.

Axelsson, P.E., 2000. DEM generation from laser scanner data using adaptive TIN models. International Archives of Photogrammetry, Remote Sensing and Spatial Information Sciences, vol. 32, no. Part B4/1, pp. 110-117. 